Case Report

\title{
Management of ameloblastic fibro-odontoma in a 6-year-old girl preserving the associated impacted permanent tooth
}

\author{
Sílvia R. A. Reis ${ }^{1)}$, Carlos E. de Freitas ${ }^{2)}$ and Alexandre R. do Espírito Santo ${ }^{3)}$ \\ ${ }^{1)}$ Department of Propaedeutics and Integrated Clinic, Federal University of Bahia School of Dentistry, \\ Salvador, BA, Brazil \\ ${ }^{2}$ Bahia's Foundation for Development of Sciences School of Dentistry, Salvador, BA, Brazil \\ ${ }^{3)}$ Department of Morphology, Piracicaba Dental School, State University of Campinas, Piracicaba, SP, Brazil
}

(Received 7 May and accepted 6 August 2007)

\begin{abstract}
The ameloblastic fibro-odontoma (AFO) is a rare odontogenic tumor that occurs predominantly in children and is generally associated with unerupted teeth. The choice of treatment for this entity is conservative surgery with enucleation and its prognosis is excellent. However, preserving the associated impacted teeth may make complete removal of the lesion difficult and may explain some cases of recurrence. A case of AFO in a 6-year-old girl treated with enucleation and preservation of an impacted lower left first permanent molar is reported. After two years of follow-up, there were no signs of recurrence and complete spontaneous eruption of the preserved tooth was observed. The present report indicates that the degree of involvement of the impacted tooth by AFO is a fundamental aspect to be considered during radiological analysis and surgical assessment. We concluded that preservation of the impacted permanent teeth associated with this tumor should always be considered and carried out with caution, since they are clearly not included in the surgical cavity. (J. Oral Sci. $49,331-335,2007$ )
\end{abstract}

Keywords: ameloblastic fibro-odontoma; odontogenic tumors; epithelium; ectomesenchyme.

Correspondence to Dr. Sílvia Regina de Almeida Reis, Departamento de Propedêutica e Clínica Integrada, Faculdade de Odontologia da UFBA, Av. Araújo Pinho, 62, Canela, Salvador, BA, CEP: 40.110-912, Brasil

Tel: +55-71-3331-0123

Fax: +55-71-3336-5976 Ext 215

E-mail: srareis@uol.com.br

\section{Introduction}

The ameloblastic fibro-odontoma (AFO) is a benign, slow-growing and expansible odontogenic tumor that consists of both odontogenic epithelium and ectomesenchyme proliferation, with dental hard tissue formation $(1,2)$. It occurs predominantly in the posterior jaws $(1,3)$ of children at a mean age of 10 years (1) and does not show significant gender predilection $(4,5)$. This tumor usually causes painless tumefaction of the affected bone and is generally associated with unerupted teeth $(1,3,4,6)$. Radiographically, the lesion presents a wellcircumscribed, unilocular or multilocular radiolucent image, and generally shows a radiopaque border at its periphery (4). The presence of radiopaque material ranging from small spots to extensive compact masses in the center of the tumor image is common $(4,7)$. Large areas of calcification make it impossible to radiographically differentiate the AFO from the complex odontoma (8). Histopathologically, the AFO is characterized by islands, strands and cords of odontogenic epithelium immersed in embryonic connective tissue that mimics primitive dental pulp $(2,4)$. Formation of osteodentin and enamel is also observed microscopically $(2,4)$. The choice of treatment for this entity is conservative surgery with enucleation and its prognosis is excellent $(2,4,5,9)$. Maintenance of impacted permanent teeth is extremely important, considering their physiological importance. However, it should always be planned and carried out with caution.

In this article, a case of AFO treated with enucleation and preservation of an associated impacted permanent first molar is reported. After two years of follow-up, there were no signs of recurrence and complete spontaneous 
eruption of the preserved tooth was observed.

\section{Case Report}

A six-year-old girl was referred to the Oral and Maxillofacial Surgery Department of the Brazilian Association of Dentistry (Salvador, Bahia, Brazil) for evaluation of the delayed eruption of the lower left permanent first molar. Extra-oral examination showed moderate facial asymmetry. On palpation, a hard and painless swelling was observed in the left mandibular body. Oral examination revealed normal colored mucosa, increase in the volume of the alveolar ridge, no definition of the vestibular fold, and absence of the lower left first molar. The panoramic radiograph exhibited a large radiopaque image enveloped by a radiolucent zone, extending from the region of the left permanent first molar to the posterior limit of mandibular body (Fig. 1). The lesion impacted the lower left first molar. The tooth germ of the lower left second molar was not detected and probably was included in the lesion. A provisional diagnosis of complex odontoma or AFO was made.

The patient underwent enucleation of the lesion and careful curettage of the surgical cavity under general anesthesia. Since the impacted tooth was clearly separated from the surgical cavity by a fibrous capsule, making sure that the entire lesion had been removed, it was decided to leave it in place and wait for its eruption. Special care was

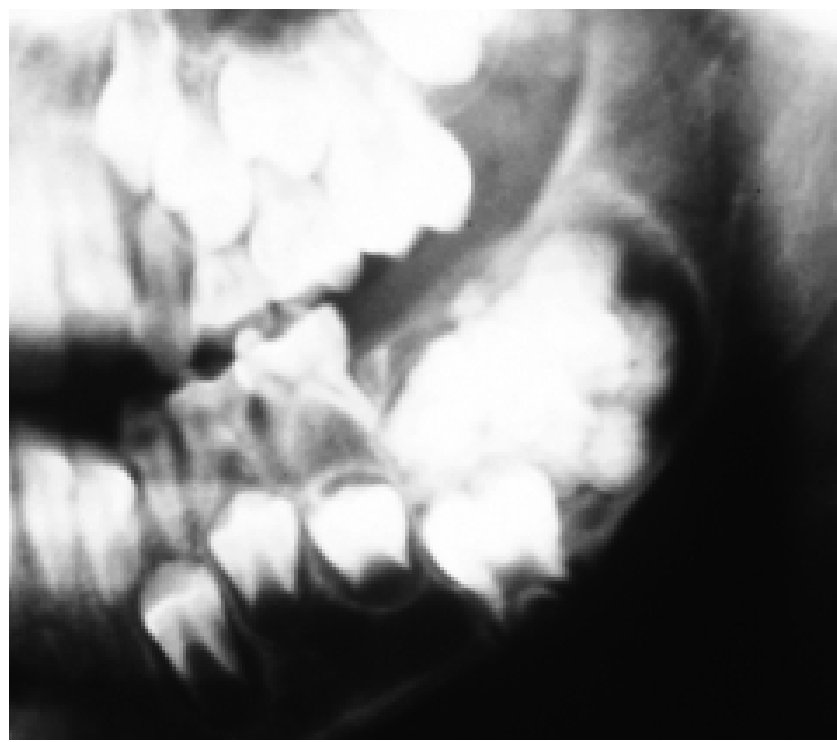

Fig. 1 Partial view of the panoramic radiograph at initial presentation. Note the large radiopaque region enveloped by a radiolucent zone, associated with the unerupted lower left permanent first molar. The germ of the lower left second molar was not identified and probably was included in the lesion. taken while performing minimum osteotomy and sectioning the relatively large calcified lesion, because of the increased risk of mandibular fracture, which is a potential complication during these procedures. Wound closure was carefully performed, especially at the periosteal level, but no specific technique (bone graft, membranes) was used to promote bone regeneration, which occurred spontaneously during the months after tumor removal.

The surgical specimen was fixed in neutral buffered $10 \%$ formalin and subjected to pathological analysis. The macroscopic exam showed a mixed lesion, presenting some areas made up of soft tissue and others of calcified material, forming a crown-like structure (Fig. 2). Upon microscopic examination, islands, strands and cords of odontogenic epithelium embedded in a cell-rich ectomesenchyme resembling dental papilla were observed (Fig. 3A). The epithelial cells were elongated showing peripheral palisading. Areas of irregular enamel and osteodentin formation were also detected (Fig. 3B). The histopathological diagnosis of AFO was made.

Follow-up clinical and radiographic examinations were carried out. Two years after surgical treatment, complete spontaneous eruption of the lower left first molar with root anomaly was observed and the germ of the lower left second molar was not noted (Fig. 4).

\section{Discussion}

The AFO is defined by the World Health Organization (WHO) as a lesion similar to ameloblastic fibroma with inductive changes that lead to the formation of dentin and enamel (4). This tumor belongs to a group of odontogenic lesions that are characterized by the simultaneous proliferation of the odontogenic epithelium and

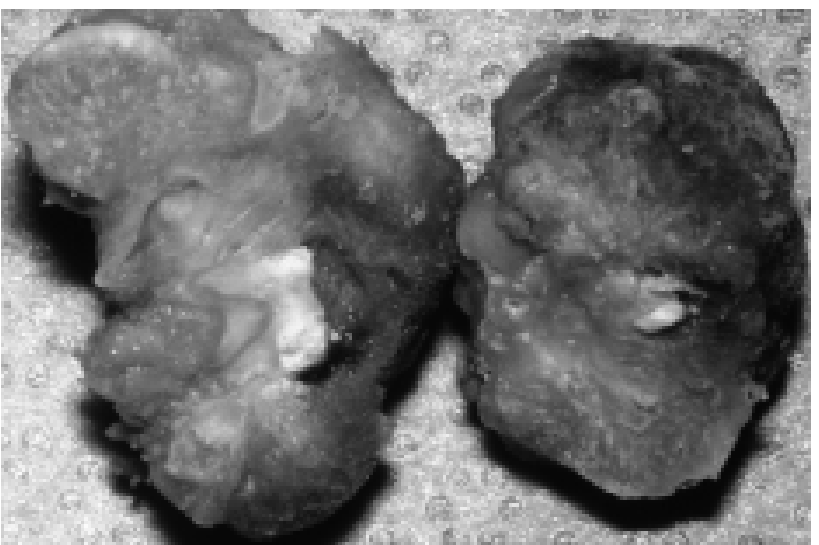

Fig. 2 Surgical specimen. Mixed lesion, presenting some areas made up of soft tissue and others of calcified material. Note the calcified tissue similar to dental crown enveloped by the tumor. 


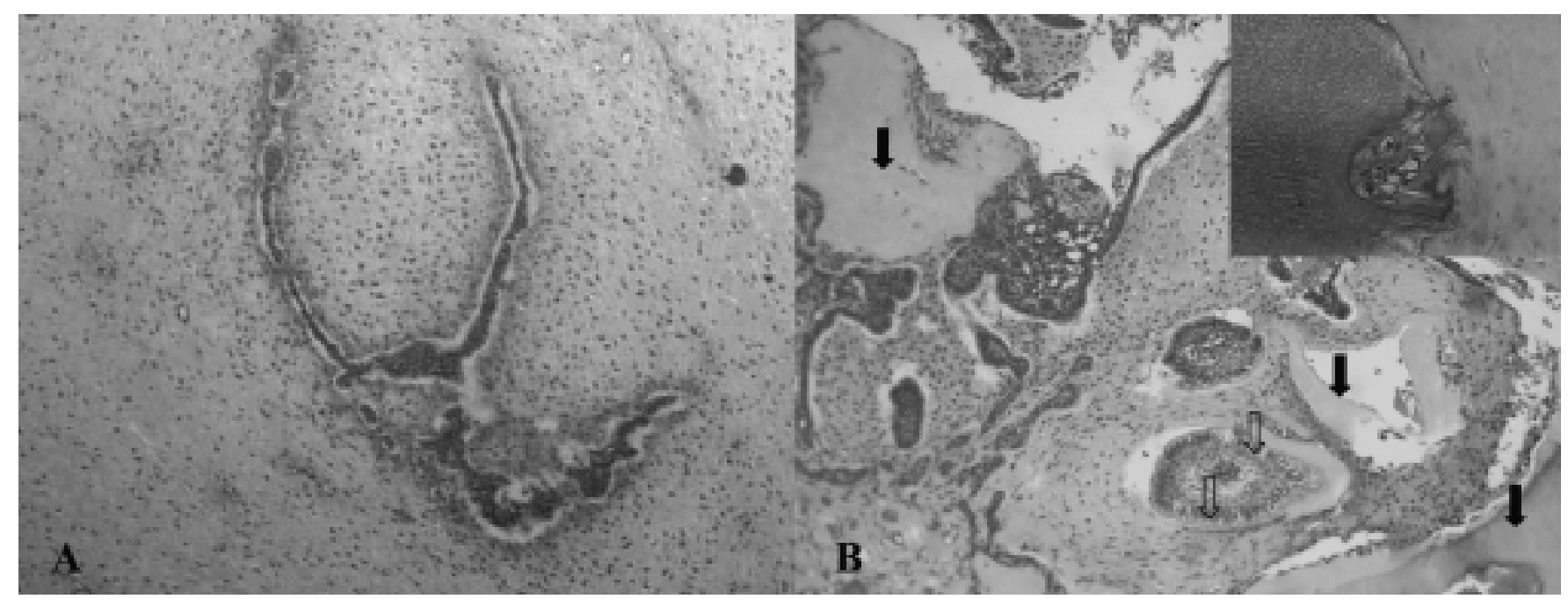

Fig. 3 Photomicrographs of the tumor. A. Cords and islands of odontogenic epithelium in a cell-rich ectomesenchyme tissue (HE stain, original magnification $\times 100$ ). B. Ectomesenchyme, odontogenic epithelium and calcified areas (full arrows). Note an epithelial island with peripheral cells similar to ameloblasts (empty arrows) (HE stain, original magnification $\times 100$ ). Note enamel matrix, osteodentin and epithelial tumoral cells in the inset (HE stain, original magnification $\times 400)$.

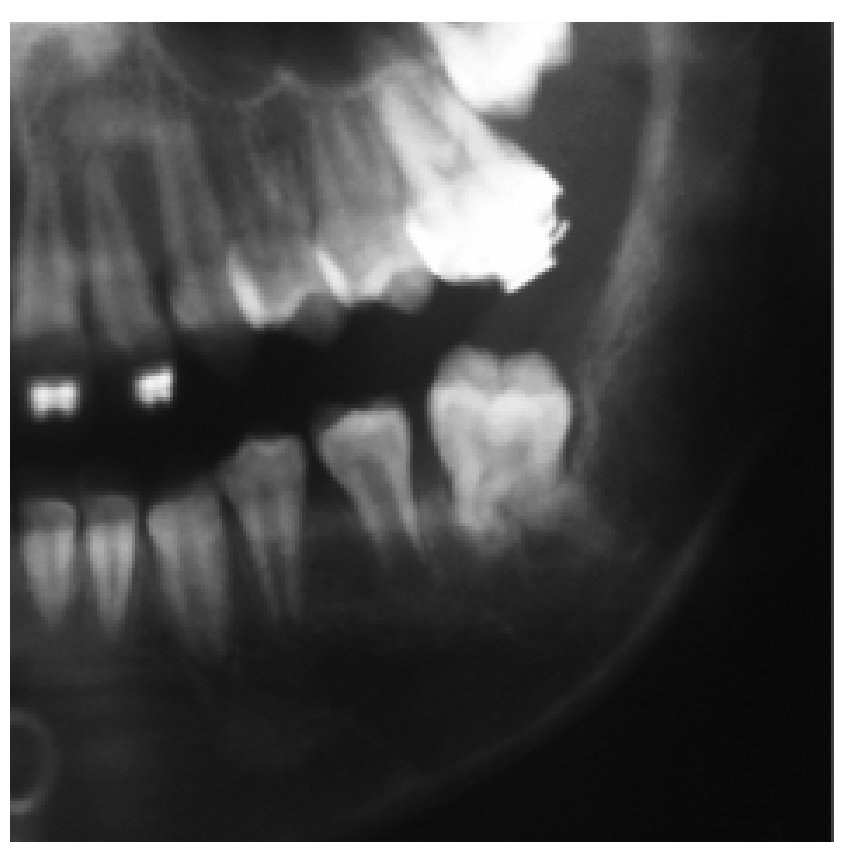

Fig. 4 Partial view of the panoramic radiograph at 2-year follow-up. There was no sign of recurrence. Complete eruption of the preserved tooth with root anomaly was observed. The germ of the lower left second molar was not noted.

ectomesenchyme (8). Ameloblastic fibroma, ameloblastic fibrodentinoma and AFO are identified as such lesions (5). The aetiology of these tumors is unknown (10). Some researchers suggest that ameloblastic fibroma and ameloblastic fibrodentinoma are precursors of AFO, which evolves into an odontoma (4,5,11). However, Slootweg (12) affirms that the ameloblastic fibroma represents a distinct neoplastic entity that does not evolve into more differentiated odontogenic lesions. It was observed that although there is no difference in prevalence between the sexes, the ameloblastic fibroma affected individuals at a higher age than the AFO did. These findings do not support the theory of precursor lesions.

The relation between AFO and the odontomas is still not clear (10). The AFO is possibly an immature complex odontoma, as the former occurs at an earlier age than the latter. Furthermore, both lesions affect similar anatomical sites (12). Philipsen, Reichart and Praetorius (10) affirmed that the AFO is a primitive stage of the hamartomatous complex odontoma. Nevertheless, the AFO should never be considered to be a hamartoma (13). The WHO has recently classified it as a tumor (4). In fact, some cases reported in the literature are truly neoplastic $(1,2,14)$ and may show malignant differentiation (9). With further regard to this discussion, Yagishita et al. (15), through immune-staining with anti-amelogenin sera, proved that tumor epithelium and mesenchyme can potentially mimic the full spectrum of phenotypic changes, and that cellular and molecular events that regulate normal odontogenesis most likely operate to a certain extent in the pathogenesis and histodifferentation of odontogenic tumors. Hence, it may be possible that these lesions would not always develop from precursor stages. Gardner (8) postulates that mixed odontogenic tumors are separate entities.

The AFO is an asymptomatic lesion with slow growth and is generally associated with an unerupted tooth (5). 
Its preferential location is the posterior jaws (5). There is no significant predilection for gender, and it usually occurs in children at a mean age of 10 years. However, this tumor has been reported in a child of less than 1 year of age (1). It tends to be well located, non-invasive, and rarely recurs after conservative surgical treatment $(8,12,16)$. Furst, Pharoah and Phillips (14) reported recurrence of an AFO treated by enucleation and preservation of the impacted tooth. In our case, 2 years after carrying out the same treatment as planned by the authors, no recurrence of this lesion was observed. A fundamental aspect to be considered with regard to the recurrence of the AFO is the degree of involvement of the impacted teeth by the tumor. Their preservation may make it difficult to completely remove the lesion, resulting in recurrence. The radiological study of the AFO reported here suggested that the impacted tooth was not included in the lesion. Surgical exposure confirmed that the tumor did not enclose the lower left first permanent molar, which was separated from the surgical cavity by a fibrous capsule. This feature contributed towards total excision of the tumor. In the panoramic radiograph taken at the initial presentation of the AFO reported by Furst, Pharoah and Phillips (14), the impacted tooth seemed to be completely included in the lesion, and total removal of the tumor was possibly difficult.

Radiographically, the AFO presents a well-circumscribed radiolucent image, containing a variable amount of radiopaque material (17). A small lesion located over an unerupted molar in a young child is most probably an immature complex odontoma, while an expansive lesion of the jaws is likely to represent an AFO (18). The presence of mineralized material is essential for diagnosis of the lesion. Nevertheless, in some cases the lesion is shown to be totally radiolucent, making it difficult to differentiate it from other odontogenic lesions. Sometimes, the mineralized component predominates in the tumor and the lesion may be radiographically similar to a complex odontoma (17). The case presented in this report had an extensive radiopaque mass, surrounded by a wellcircumscribed radiolucent zone (Fig. 1). In lesions with large amounts of mineralized material, histological differentiation from the complex odontoma is difficult (2). The AFO is made up of islands, strands and cords of odontogenic epithelium immersed in a cell-rich embryonic connective tissue, containing a variable amount of irregular formation of enamel and osteodentin $(4,5,19)$. In spite of the large radiopaque mass observed in the present case, proliferation of ectomesenchymal component containing strands and islands of odontogenic epithelium was found. The abovementioned characteristics, the large extent of the lesion, and the significant bone expansion were fundamental for differentiating it from a complex odontoma. Odontoameloblastoma may be considered as a differential diagnosis. However, the clinical and histopathological features excluded this diagnosis.

The conservative surgical treatment performed in this case was carefully carried out in order to remove the lesion completely, and to maintain the integrity of the lower left first molar germ. Two years after surgery, complete eruption of the tooth occurred without any orthodontic treatment. However, dilaceration of the roots was observed. Even with this anomaly, the preservation of the tooth referred to represents the great success of the chosen treatment, considering the physiological importance of the permanent first molar. The tooth germ of the lower left second molar was not observed and was probably related to the genesis of the tumor. To conclude, the impacted teeth associated with AFO should always be preserved, since they are clearly not included in the surgical cavity.

\section{Acknowledgments}

We thank Dr. Jorge Esquiche Léon (DDS, MS, PhD Student, Department of Oral Diagnosis, Piracicaba Dental School, State University of Campinas, São Paulo, Brazil) for critical reading of the manuscript.

\section{References}

1. Baker WR, Swift JQ (1993) Ameloblastic fibroodontoma of the anterior maxilla. Report of a case. Oral Surg Oral Med Oral Pathol 76, 294-297

2. Friedrich RE, Siegert J, Donath K, Jäkel KT (2001) Recurrent ameloblastic fibro-odontoma in a 10year-old boy. J Oral Maxillofac Surg 59, 1362-1366

3. Favia GF, Di Alberti L, Scarano A, Piattelli A (1997) Ameloblastic fibro-odontoma: report of two cases. Oral Oncol 33, 444-446

4. Barnes L, Eveson JW, Reichart P, Sidransky D (2005) World Health Organization classification of tumours. Pathology and genetics of head and neck tumours. IARC Press, Lyon, 309

5. Waldron CA (1995) Odontogenic cysts and tumors. In Oral and maxillofacial pathology, Neville BW, Damm DD, Allen CM, Bouquot JE eds, WB Saunders, Philadelphia, 493-511

6. Villarreal PM, Junquera LM, Albertos JM, Molina R, Gonzalez S (1998) Ameloblastic fibro-odontoma. Clinical aspects and review of the literature. Rev Stomatol Chir Maxillofac 98, 349-353 (in French)

7. Chang H, Precious DS, Shimizu MS (2002) Ameloblastic fibro-odontoma: a case report. J Can Dent Assoc 68, 243-246

8. Gardner DG (1984) The mixed odontogenic tumors. 
Oral Surg Oral Med Oral Pathol 58, 166-168

9. Howell RM, Burkes EJ Jr (1977) Malignant transformation of ameloblastic fibro-odontoma to ameloblastic fibrosarcoma. Oral Surg Oral Med Oral Pathol 43, 391-401

10. Philipsen HP, Reichart PA, Praetorius F (1997) Mixed odontogenic tumors and odontomas. Considerations on interrelationship. Review of the literature and presentation of 134 new cases of odontomas. Oral Oncol 33, 86-99

11. Miller AS, López CF, Pullon PA, Elzay RP (1976) Ameloblastic fibro-odontoma. Report of seven cases. Oral Surg Oral Med Oral Pathol 41, 354-365

12. Slootweg PJ (1981) An analysis of the interrelationship of the mixed odontogenic tumors - ameloblastic fibroma, ameloblastic fibroodontoma, and the odontomas. Oral Surg Oral Med Oral Pathol 51, 266-276

13. Takeda Y (1999) Ameloblastic fibroma and related lesions: current pathologic concept. Oral Oncol 35, 535-540

14. Furst I, Pharoah M, Phillips J (1999) Recurrence of an ameloblastic fibro-odontoma in a 9-year-old boy. J Oral Maxillofac Surg 57, 620-623

15. Yagishita H, Taya Y, Kanri Y, Matsuo A, Nonaka H, Fujita H, Aoba T (2001) The secretion of amelogenins is associated with the induction of enamel and dentinoid in an ameloblastic fibroodontoma. J Oral Pathol Med 30, 499-503

16. Okura M, Nakahara H, Matsuya T (1992) Treatment of ameloblastic fibro-odontoma without removal of the associated impacted permanent tooth: report of cases. J Oral Maxillofac Surg 50, 1094-1097

17. Langlais RP, Langland OE, Nortje CJ (1995) Diagnostic imaging of the jaws. Williams \& Wilkins, Baltimore, 315-318

18. Miyauchi M, Takata T, Ogawa I, Ito H, Nikai H, Ijuhin N, Tanimoto K, Miyauchi S (1996) Immunohistochemical observations on a possible ameloblastic fibro-odontoma. J Oral Pathol Med 25, 93-96

19. al-Sebaei MO, Gagari E (2001) Ameloblastic fibroodontoma. J Mass Dent Soc 50, 52-53 